\title{
ANIMAL EXPERIMENTATION
}

\section{Study of the In Vitro Maturation of Mouse Oocytes Induced by Microinjection of Maturation Promoting Factor (MPF)}

\author{
HIDEYUKI NAKANO $^{1}$ and HARUMI KUBO ${ }^{1}$
}

Submitted: March 25, 1999

Accepted: June 30, 1999

\begin{abstract}
Purpose: Maturation promoting factor (MPF) acts at the resumption of meiosis and nonspecifically throughout the animal species. There exists a considerable body of literature on MPF, but little work has been done to study the induction of maturation of mammalian oocytes by microinjection of extracted $M P F$
\end{abstract}

Methods: Immature ( $G V$-stage) mouse oocytes were microinjected MPF extracted from matured Xenopus eggs in the presense of dbcAMP.

Results: The rate of germinal vesicle breakdown (GVBD) induced at 24 hr after MPF injection was significantly higher $(90.5 \%)$ than that of the control $(2.2 \%)$, which was injected with HTF medium containing dbcAMP $(P<0.0001)$. The rate of extrusion of the first polar body at $24 \mathrm{hr}$ after $M P F$ injection was significantly higher (84.1\%) than that of the same control $(1.1 \%)(P<0.0001)$.

Conclusions: From these results, it is concluded that the maturation of mammalian oocytes can be induced by the microinjection of MPF extracted from other species.

KEY WORDS: maturation promoting factor; immature oocyte; in vitro maturation; microinjection.

\section{INTRODUCTION}

In most animals, fully grown oocytes in the ovary are naturally arrested at prophase of the first meiotic division, and meiosis is resumed by cytoplasmic activation. Then oocytes proceed through meiosis II, arresting at metaphase as unfertilized eggs. Meiotic maturation is necessary for the oocytes to be fertilized

\footnotetext{
${ }^{1}$ First Department of Obstetrics and Gynecology, Toho University School of Medicine; 6-11-1, Ohmori-nishi, Ohta Word, Tokyo, 143, Japan.
}

normally. These immature oocytes have a large nucleus called a germinal vesicle and are competent to resume meiosis. The resumption of meiosis is morphologically identified by germinal vesicle breakdown (GVBD) and, subsequently, by chromosome condensation, spindle formation, and first polar body emission.

Recently, maturation promoting factor (MPF) has been shown to be an important regulator of the cell cycle during the process of oocyte maturation. MPF was first described in 1971 as a cytoplasmic activity which appeared during meiosis in amphibian oocytes (1). MPF acts at the resumption of both the meiotic and the mitotic phases in a general, nonspecific manner in all animals (2). The presence of MPF in mammalian oocytes has been convincingly demonstrated by the microinjection of the rabbit, mouse, and pig maturing cytoplasm into immature oocytes of the same species, and the microinjection of maturing mouse oocyte cytoplasm into the immature oocytes of starfish, amphibian, and pig (3-10).

MPF was first extracted and purified in 1988 (11). However, there is still little known about the mechanism of induction of maturation of mammalian eggs by microinjection of extracted MPF. In this study, we partially purified MPF from mature Xenopus oocytes and observed GVBD and first polar body formation after the microinjection of MPF into mouse immature (GV-stage) oocytes.

\section{MATERIALS AND METHODS}

\section{Purification of MPF $(11,12)$}

Xenopus laevis females were injected with $100 \mathrm{IU}$ pregnant mare serum gonadotropin (PMSG; Teikoku 
Zoki, Tokyo) $96 \mathrm{hr}$ before an injection of $750 \mathrm{IU}$ human chorionic gonadotropin (hCG; Teikoku Zoki, Tokyo). In the evening before our experiments, they were injected with hCG and placed in tap water containing $0.1 M \mathrm{NaCl}$. Eggs were collected, dejelled in $2 \%$ cysteine, and washed well with $50 \mathrm{mM}$ Tris- $\mathrm{HCl}$.

Dejelled Xenopus eggs were washed with improved MPF extraction buffer consisting of $15 \mathrm{mM} \mathrm{MgCl} 2$, $20 \mathrm{~m} M$ HEPES, $80 \mathrm{~m} M \beta$-glycerophosphate, $20 \mathrm{~m} M$ EGTA, $50 \mathrm{~m} M \mathrm{NaF}, 1 \mathrm{~m} M$ dithiothreitol (DTT), $20 \mathrm{~m} M$ $\alpha$-naphthyl phosphate, $0.3 \mathrm{~m} M$ phenylmethylsulfonyl fluoride (PMSF), $3 \mu \mathrm{g} / \mathrm{ml}$ leupeptin, and $1 \mathrm{~m} M$ ATP$\gamma \mathrm{s}$. Eggs were crushed by centrifugation at $15,000 \mathrm{~g}$ for $15 \mathrm{~min}$. The material between the lipid cap and the pellet was centrifuged at $100,000 \mathrm{~g}$ for $4 \mathrm{hr}$ after the addition of $3.8 \mathrm{M}\left(\mathrm{NH}_{4}\right)_{2} \mathrm{SO}_{4}$.

After removal of the supernatant, the precipitate was suspended in dialysis buffer (DB: $100 \mathrm{~m} M \beta$ glycerophosphate, $15 \mathrm{~m} M \mathrm{MgCl}_{2}, 5 \mathrm{~m} M$ EGTA, 20 $\mathrm{m} M$ HEPES, $1 \mathrm{~m} M$ DTT, $0.3 \mathrm{~m} M$ PMSF), dialyzed in DB for $12 \mathrm{hr}$, and stored at $-80^{\circ} \mathrm{C}$.

\section{Preparation of Demembranated Sperm Nuclei}

Testes were collected from male Xenopus laevis and broken into pieces. After centrifugation with $70 \%$ Percoll solution at $5000 \mathrm{~g}$ for $10 \mathrm{~min}$, sucrose buffer (SB) consisting of $0.2 \mathrm{M}$ sucrose, $2.5 \mathrm{mM} \mathrm{MgCl}$, and $10 \mathrm{~m} M$ HEPES was added, and the mixture centrifuged at $5000 \mathrm{~g}$ for $15 \mathrm{~min}$. The supernatant was removed, and $0.5 \mathrm{mg} / \mathrm{ml}$ lisolecitine and $100 \mu \mathrm{g} / \mathrm{ml}$ leupeptin were added to the pellet. After the addition of SB containing $3 \%$ bovine serum albumin (BSA), the material was centrifuged at $5000 \mathrm{~g}$ for $15 \mathrm{~min}$. The demembranated sperm nuclei were centrifuged at $5000 \mathrm{~g}$ for 15 min after the addition of $0.4 \%$ BSA.

\section{Preparation of Extracts Causing Pronuclear Formation}

Dejelled eggs were washed in pronucleus extraction buffer consisting of $0.1 \mathrm{M} \mathrm{KCl}, 5 \mathrm{mM} \mathrm{MgCl} 2,20 \mathrm{~m} M$ HEPES, $2 \mathrm{~m} M \beta$-mercaptoethanol, $0.3 \mathrm{~m} M$ PMSF, and $3 \mu \mathrm{g} / \mathrm{ml}$ leupeptin and were crushed by centrifugation at $15,000 \mathrm{~g}$ for $15 \mathrm{~min}$. The lipid cap and the pellet were removed, and cytochalasin B was added to a final concentration of $50 \mu \mathrm{g} / \mathrm{ml}$. The extract was centrifuged again at $15,000 \mathrm{~g}$ for $15 \mathrm{~min}$, and the supernatent was incubated with the nuclei for $1 \mathrm{hr}$ at $19^{\circ} \mathrm{C}$.

\section{Assay of MPF Activity in a Cell-Free System}

MPF activity was assayed by serial dilution with $100 \mathrm{~m} M$ sodium-glycerophosphate, $20 \mathrm{~m} M$ HEPES, $15 \mathrm{~m} M \mathrm{MgCl}_{2}, 5 \mathrm{~m} M$ EGTA, and $1 \mathrm{~m} M$ DTT. It was defined as the minimum amount required to cause nuclear envelope breakdown and chromosome condensation after the incubation. We assayed the same sample four times. A 6- $\mu$ l aliquot of extract was added to the same amount of the sample, and the mixture was incubated for $2 \mathrm{hr}$ at $19^{\circ} \mathrm{C}$. It was mixed with an equal volume of a $4^{\prime}, 6^{\prime}$-diamidine-2-phenylindole solution on a glass slide and observed with an opiphotomicroscope.

\section{Collection and Culture of Immature Mouse Oocytes}

ICR female mice 8-10 weeks old were induced to superovulate by intraperitoneal injections of $5 \mathrm{IU}$ PMSG 42-48 hr before the removal of their ovaries. Mice were kept in the light for $12 \mathrm{hr}$ and in the dark for $12 \mathrm{hr}$.

We used several kinds of medium: HTF medium (Irvine Scientific, USA), HTF medium supplemented with $150 \mu \mathrm{g} / \mathrm{ml}$ dibutyryl cyclic adenosine-3' $5^{\prime}$-monophosphate (dbcAMP) (cAMP-HTF medium), HTF medium containing $21.0 \mathrm{~m} M$ HEPES (mHTF medium; Irvine Scientific), and mHTF medium supplemented with $150 \mu \mathrm{g} / \mathrm{ml} \mathrm{dbcAMP} \mathrm{(cAMP-mHTF} \mathrm{medium).}$ These were supplemented with $3 \mathrm{mg} / \mathrm{ml} \mathrm{BSA}$. Oocytes were cultured at $37^{\circ} \mathrm{C}$ in a humidified atmosphere of $5 \% \mathrm{CO}_{2}$ in air.

The mice were sacrificed by cervical dislocation and the ovaries were removed in cAMP-HTF medium. The preovulatory large antral follicles were punctured with fine forceps in cAMP-HTF medium under a light microscope. Immature oocytes with cumulus cells were collected in the same medium. Cumulus cells were removed from oocytes by gently repeated pipetting of the cumulus-oocyte complex through finely drawn pipettes, washed several times, and pooled in cAMP-HTF medium.

A total of 100 oocytes was used in five separate experiments. For all study groups, morphologically normal oocytes were those containing a fully grown germinal vesicle $(\mathrm{GV})$ in the center of the cytoplasm, and with a uniform color and distribution of the cytoplasm. GVBD and degenerated oocytes were excluded from the experiment.

Mouse GV-stage oocytes obtained in cAMP-HTF medium were left in cAMP-mHTF medium under min- 
eral oil for $25 \mathrm{~min}$ at $17-18^{\circ} \mathrm{C}$ and for $10 \mathrm{~min}$ at room temperature. Immediately after that, the oocytes were cultured in either HTF medium (HTF group) or cAMP$\mathrm{HTF}$ medium (cAMP group) in a $\mathrm{CO}_{2}$ incubator $\left(37^{\circ} \mathrm{C}\right.$, $5 \% \mathrm{CO}_{2}, 95 \%$ air) for $24 \mathrm{hr}$. At various times during incubation, oocytes were examined under a phasecontrast microscope for GVBD and emission of the first polar body.

\section{Microinjection with cAMP-mHTF Medium and MPF (Fig. 1) (13)}

The outer and inner diameters of micropipettes were 75 and $15 \mu \mathrm{m}$ for holding, respectively, and 5 and 2-3 $\mu \mathrm{m}$ for injection, respectively. The movements of the micropipettes were controlled by Narishige NT88 micromanipulators that were mounted on a Nikon inverted microscope (TMD300-2).

Oocytes were immobilized by the holding pipette in the 9 o'clock position, and the injection pipette was

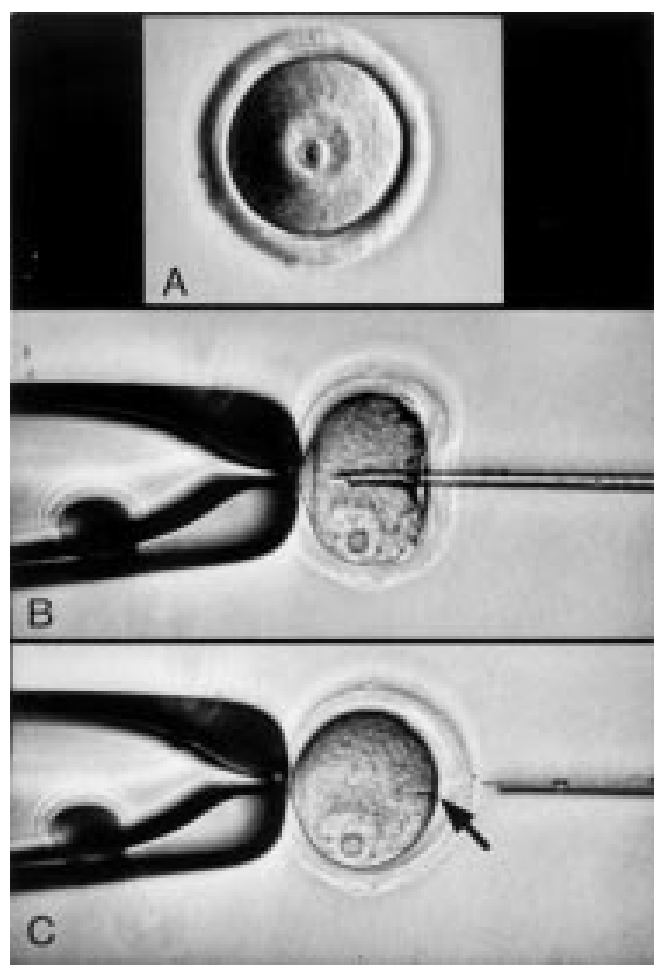

Fig. 1. Microinjection of MPF into an immature (GV-stage) mouse oocytes. (A) One hundred morphologically normal oocytes were used for all study groups. (B) Oocytes were immobilized by the holding pipette in the 9 o'clock position, and the injection pipette was inserted through the zona pellucida in the 3 o'clock position deep into the ooplasm to avoid injury to the GV. (C) The oocytes were injected with $10-20 \mathrm{pl}$ of medium or MPF in cAMP-mHTF medium under mineral oil. inserted through the zona pellucida in the 3 o'clock position, deep into the ooplasm to avoid injury to the GV. The oocytes were injected with $10-20 \mathrm{pl}$ of cAMP-mHTF medium (cAMP-mHTF group) or MPF (MPF group) in cAMP-mHTF medium under mineral oil at $17-18^{\circ} \mathrm{C}$ in the atmosphere. After microinjection, oocytes were left in cAMP-mHTF medium under mineral oil for $25 \mathrm{~min}$ at $17-18^{\circ} \mathrm{C}$ and $10 \mathrm{~min}$ at room temperature. Immediately after that, the oocytes were cultured in cAMP-HTF medium in a $\mathrm{CO}_{2}$ incubator $\left(37^{\circ} \mathrm{C}, 5 \% \mathrm{CO}_{2}, 95 \%\right.$ air $)$ for $24 \mathrm{hr}$. At various times during incubation, oocytes were examined under a phase-contrast microscope for GVBD and emission of the first polar body.

For statistical analysis Student's $t$ test was used to determine significance.

\section{RESULTS}

\section{Assay of MPF Activity}

MPF activity became evident with the induction of chromosome condensation and nuclear envelope breakdown, and MPF inactivity became evident with the induction of nuclear envelope assembly and chromosome decondensation in the demembranated Xenopus sperm nuclei (Fig. 2). The activity was decided after $32 \times$ dilution (Table I).

\section{In Vitro Spontaneous Maturation of Mouse GV- Stage Oocytes (HTF Group)}

This experiment was undertaken to determine the in vitro maturation of mouse GV-stage oocytes removed from the ovaries. The results are presented in Figs. 2 and 3. About half of the GV-stage oocytes underwent GVBD at $1 \mathrm{hr}(48.0 \%)$. After that, the percentage increased gradually, and $95.0 \%$ of the oocytes had undergone GVBD by $24 \mathrm{hr}$ (Fig. 3). They began to extrude the first polar body within $3-4 \mathrm{hr}(8.1 \%$ at 4 $\mathrm{hr}$ ) and had increased suddenly at 5-6 hr. At $24 \mathrm{hr}$, $74.6 \%$ of oocytes had undergone first polar body formation (Fig. 4). The degeneration rate of the oocytes was $2.0 \%$ at $24 \mathrm{hr}$.

\section{Inhibitory Effect of dbcAMP on GVBD and First Polar Body Formation of Mouse GV-Stage Oocytes (cAMP group)}

This experiment was designed to confirm that dbcAMP inhibited spontaneous maturation of mouse 

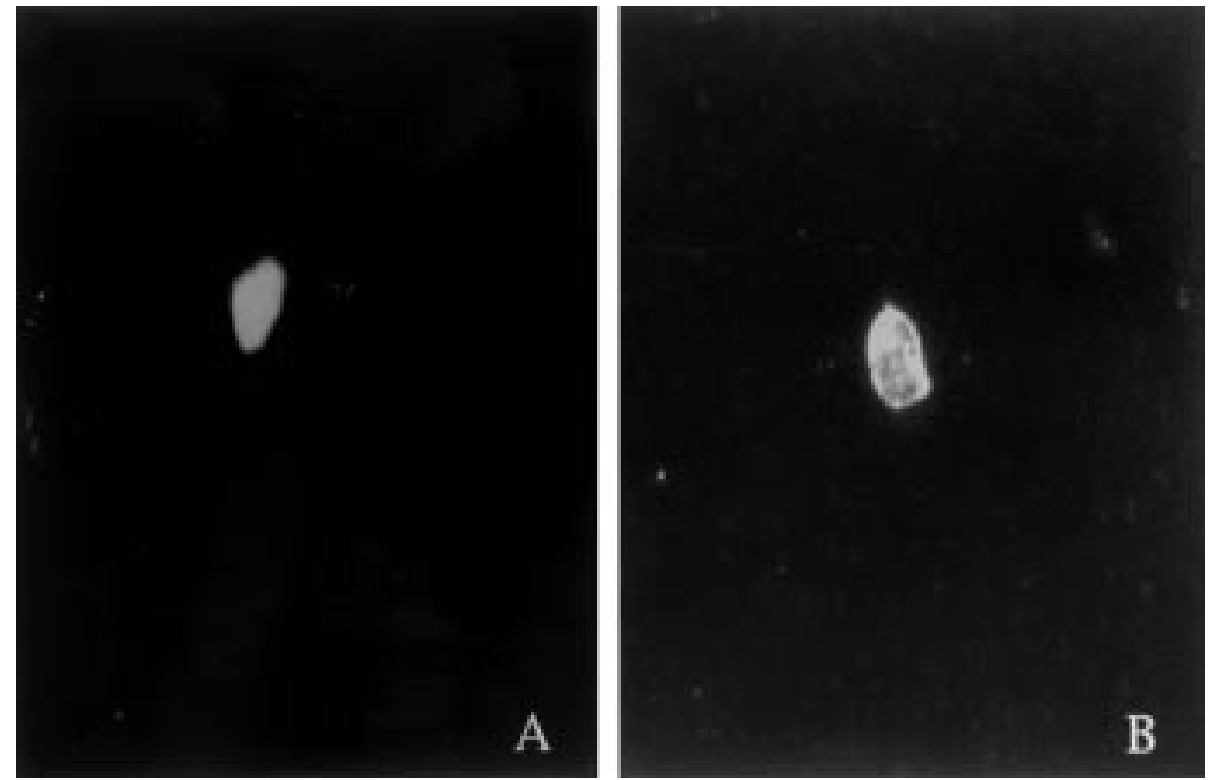

Fig. 2. Sperm nuclei of Xenopus laevis to assay MPF activity in a cell-free system. MPF activity became evident with the induction of chromosome condensation and nuclear envelope breakdown (A), and MPF inactivity became evident with the induction of nuclear envelope assembly and chromosome decondensation (B) to the demembranated Xenopus sperm nuclei after assay of MPF activity.

GV-stage oocytes (Figs. 3 and 4). The GV-stage oocytes underwent low-level GVBD (3.1\% at $24 \mathrm{hr})$ and extruded their first polar body within $24 \mathrm{hr}(1.1 \%$ at $24 \mathrm{hr}$ ).

At all time points, the percentage showing GVBD and first polar body formation was significantly higher for the HTF group compared with the cAMP group $(P<0.0001)$ (Figs. 3 and 4$)$. The degeneration rate of the oocytes was $3.0 \%$ at $24 \mathrm{hr}$. There was no significant difference between the HTF and the cAMP group.

\section{Effect of dbcAMP on GVBD and First Polar Body Formation of Mouse GV-Stage Oocytes Injected with Medium Containing dbcAMP (cAMP-Med Group)}

This experiment was undertaken to determine whether the GV-stage oocytes matured spontaneously

Table I. Activity of Crude $\mathrm{MPF}^{a}$

\begin{tabular}{ccccccccc}
\hline $\begin{array}{c}\text { Expt } \\
\text { No. }\end{array}$ & 1 & 2 & 4 & 8 & 16 & 32 & 64 & Control \\
\hline 1 & + & + & + & + & + & + & - & - \\
2 & + & + & + & + & + & + & - & - \\
3 & + & + & + & + & + & + & - & - \\
4 & + & + & + & + & + & + & - & - \\
\hline
\end{tabular}

a Activity was assayed by serial dilution. It was defined as the minimum amount that caused nuclear envelope breakdown and chromosome condensation after incubation. We assayed each example, four times. (+) activity present $(-)$ activity absent. through micromanipulation. The GV-stage oocytes hardly underwent GVBD (2.2\% at $24 \mathrm{hr})$ and extruded their first polar body within $24 \mathrm{hr}(1.1 \%$ at $24 \mathrm{hr})$ (Figs. 3 and 4). The degeneration rate of the injected oocytes was $6.1 \% 24 \mathrm{hr}$ after microinjection.

\section{In Vitro Maturation of Mouse GV-Stage Oocytes Injected with MPF (MPF Group)}

In a preliminary experiment, 30 oocytes were injected with Buffer A. However, no immature oocytes were induced to undergo GVBD or first polar body formation.

The effect of microinjection with MPF extracted from unfertilized Xenopus eggs on GV-stage oocytes maturation in vitro was then examined. Oocytes began to undergo GVBD at $1 \mathrm{hr}$ after microinjection (44.4\% at $1 \mathrm{hr}$ ). After that the rate rapidly increased between 2 and $6 \mathrm{hrs}$, and $90.5 \%$ of the oocytes underwent GVBD at $24 \mathrm{hr}$ after microinjection (Fig. 3). They began to extrude the first polar body within $2-3 \mathrm{hr}$ after microinjection. After that the rate increased suddenly at $5-6 \mathrm{hr}$, and $84.1 \%$ of the oocytes had extruded their first polar body at $24 \mathrm{hr}$ after microinjection (Fig. 4). At all time points, the percentage showing GVBD and first polar body formation was significantly higher for the MPF group compared with the cAMP-Med group $(P<0.0001)$. The degeneration rate of the injected oocytes was $6.0 \%$ at $24 \mathrm{hr}$ after microinjection. 


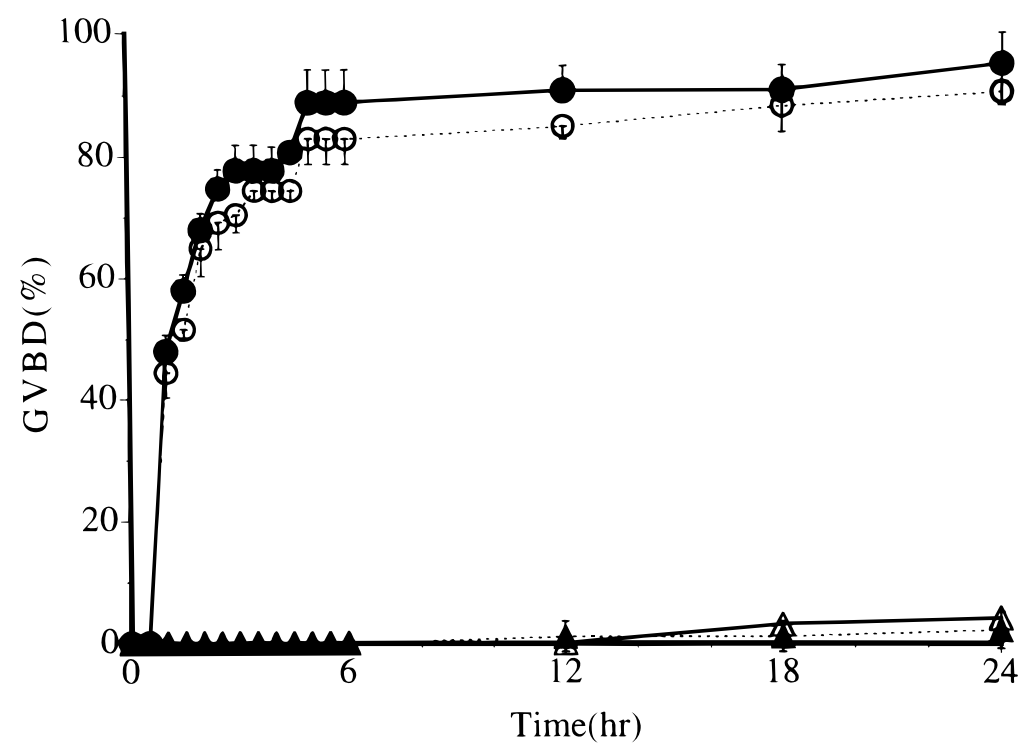

Fig. 3. Effect on GVBD time course of MPF microinjection into mouse GV-stage oocytes. Oocytes were incubated in the presence $(\triangle)$ or absence $(-)$ of dbcAMP $(150 \mu \mathrm{g} / \mathrm{ml})$. Oocytes were microinjected with $10-20 \mathrm{pl}$ of either a solution of MPF (O) or a control solution $(\boldsymbol{\Lambda})$ in the presence of $\operatorname{dbcAMP}(150 \mu \mathrm{g} / \mathrm{ml})$. The experiment was repeated five times, and the number of oocytes examined in each group was 100 .

There was no significant difference between the MPF and the cAMP-Med groups.

There was no significant difference between the GVBD and the degeneration rate at any time point between the MPF and the HTF groups. There was also no significant difference between these groups in first polar body formation rate within $3 \mathrm{hr}$, but from $4 \mathrm{hr}$ the rate in the MPF group was significantly higher

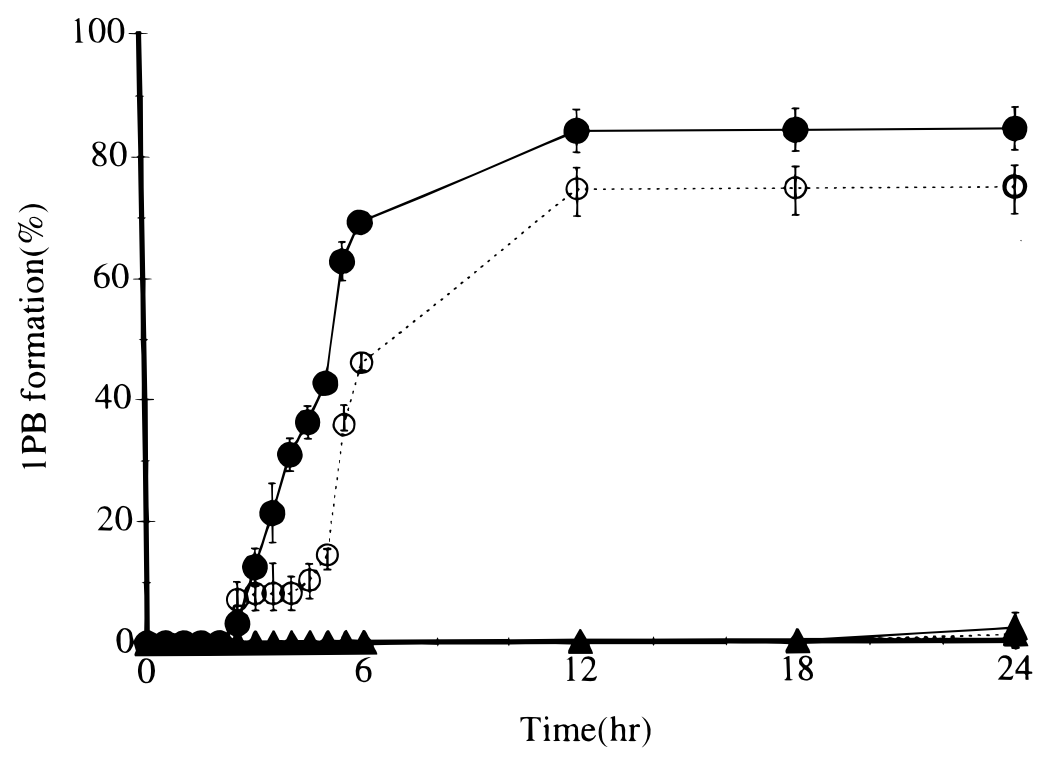

Fig. 4. Effect on time course of first polar body (1PB) formation of MPF microinjection into mouse GV-stage oocytes. Oocytes were incubated in the presence $(\triangle)$ or absence $(-)$ of dbcAMP $(150 \mu \mathrm{g} / \mathrm{ml})$. Oocytes were microinjected with $10-20 \mathrm{pl}$ of either a solution of MPF $(\bigcirc)$ or a control solution $(\mathbf{\Lambda})$ in the presence of dbcAMP $(150 \mu \mathrm{g} / \mathrm{ml})$. The experiment was repeated five times, and the number of oocytes examined in each group was 100 . 
than that in the HTF group $(P<0.001$ within $6 \mathrm{hr}$ and $P<0.005$ between 12 and $24 \mathrm{hr}$ ).

\section{DISCUSSION}

In the process of the oocyte maturation, the mechanism of regulation of release from prophase arrest in fully grown immature oocytes is species-specific. The onset of oocyte maturation is triggered by maturationinducing substance (MIS), which is found in follicle cells surrounding oocytes in the ovary. MIS has been identified as 1-methyladenine in starfish and progesterone in amphibians but has not been identified in mammalian oocytes. MIS is produced by follicle cells following stimulation by gonadotropins and acts directly on the immature oocyte surface to produce a cytoplasmic factor called MPF. MPF induces GVBD and subsequent maturational events within oocytes, and its activity changes through the meiotic cycle: MPF reaches a peak at the first metaphase and disappears at the first polar body emission. Then MPF reappears and reaches a peak again at the second metaphase. Oocyte maturation is arrested again at the second metaphase in vertebrate animals, but this arrest is released by fertilization.

The resumption of oocyte maturation from the first metaphase can be inhibited by oocyte maturation inhibitor (OMI), which has a molecular weight of $2 \mathrm{kD}$. OMI is released from granulosa cells, and OMI acts on the cumulus-oocyte complex. The inhibition of oocyte maturation by OMI is reversible and is released by the inhibition of OMI (14).

The inhibition of in vitro maturation of immature oocytes by cyclic AMP (cAMP) and phosphodiesterase inhibitor has been extensively investigated. Cho et al. reported that $100 \mu \mathrm{g} / \mathrm{ml}$ dibutyryl cAMP (dbcAMP) inhibited the in vitro maturation of mouse immature oocytes (15). We confirmed the inhibition of spontaneous oocyte maturation by cAMP. GV-stage oocytes underwent low-level GVBD (3.1\% at $24 \mathrm{hr}$ ) and extrusion of their first polar body (1.1\% at $24 \mathrm{hr})$. However, Lindner et al. reported that the intracellular levels of cAMP in the immature mouse oocytes increased and the oocytes matured when the oocytes were liberated from antral follicles and cultured in the presence of LH (16). They could induce oocyte maturation by injecting dbcAMP into the follicular fluid in the absence of LH. Dekel et al. found that inhibition of maturation of rat oocytes was obtained by a relatively low dose of dbcAMP, while higher concentrations of
dbcAMP were required to induce oocyte maturation (17).

While each MIS is species-specific, MPF acts in a non-species-specific manner and appears to play a key role in promoting M-phase within the cytoplasm of all eukaryotic cells regardless of whether they are undergoing meiosis or mitosis (2).

In almost all mammals, immature oocytes are released from prophase arrest when they are cultured in vitro. Mouse immature oocytes underwent GVBD spontaneously after 8 to $10 \mathrm{hr}$ of incubation and most oocytes have emitted the first polar body by 16 to 24 $\mathrm{hr}$ (18). In the present study, $95.0 \%$ of mouse immature oocytes underwent GVBD spontaneously and $74.6 \%$ of oocytes emitted the first polar body after $24 \mathrm{hr}$ of incubation.

In mammalian oocytes, the presence of MPF has been found by the transfer of MPF from donor cell to recipient cell between different species using microinjection techniques $(8-10)$. However, few studies have examined the microinjection of purified or partly purified MPF. In the present study, we observed GVBD and first polar body formation after the microinjection of MPF into mouse immature oocytes treated with cAMP to inhibit spontaneous maturation. Our results suggest that the resumption of prophase arrest was induced by injection of MPF extracted from Xenopus mature oocytes, however, it is possible that the maturation was induced not only by the exogenous MPF of Xenopus oocytes but also by the endogenous MPF of mouse oocytes. However, maturation was not due to physical manipulation or the phosphodiesterase-containing Buffer A, because it was not induced by microinjection with HTF medium supplemented with dbcAMP and Buffer A (19).

From our results, there is the possibility that the maturation of mammalian oocytes can be induced by the microinjection of MPF extracted from other species. Furthermore, it is possible that we might be able to apply MPF to assisted reproductive technology if MPF were purified much more and we solved many problems, for example, ethical issues and DNA contamination of another species.

\section{ACKNOWLEDGMENTS}

We thank Prof. Shigeru Yamashita, Department of Biochemistry, Toho University School of Medicine, for technical assistance, Prof. Shun Hirakawa, First Department of Obstetrics and Gynecology, Toho University School of Medicine, for valuable discussion, 
and Prof. Hisao Amano, Department of Biochemistry, Toho University School of Medicine, for advice and support.

\section{REFERENCES}

1. Masui Y, Markert CL: Cytoplasmic control of nuclear behavior during meiotic maturation of frog oocytes. J Exp Zool 1971; 177:129-146

2. Kishimoto T: Regulation of metaphase by a maturation-promoting factor. Dev Growth Different 1988:30:105-115

3. Hashimoto N, Kishimoto T: Regulation of miotic metaphse by a cytoplasmic maturation-promoting factor during mouse oocyte maturation. Dev Biol 1988; 126:242-252

4. Sorensen RA, Cyert MS, Pedersen RA: Active maturationpromoting factor is present in mature mouse oocytes. J Cell Biol 1985;100:1637-1640

5. Miake-Lye R, Newport J, Kirschner M: Maturation-promoting factor induces nuclear envelope breakdown in cycloheximidearrested embryos of Xenopus laevis. J Cell Biol 1983;97:81-91

6. Balakier H, Czolowska R: Cytoplasmic control of nuclear maturation in mouse oocytes. Exp Cell Res 1977;110:466-469

7. Fulka J Jr: Nuclear maturation in pig and rabbit oocytes after interspecific fusion. Exp Cell Res 1983;146:212-218

8. Prochazka R, Motlic J, Fulka J: Activity of maturation promoting factor in pig oocytes after microinjection and serial transfer of maturing cytoplasm. Cell Different Dev 1989;27:175-182

9. Kishimoto T, Yamazaki K, Kato Y, et al.: Induction of starfish oocyte maturation by maturation-prommoting factor of mouse and surf clam oocytes. J Exp Zool 1984;231:293-295
10. Sunkara PS, Wright DA, Rao PN: Mitotic factors from mammalian cells induce germinal vesicle breakdown and chromosome condensation in amphibian oocytes. Proc Natl Acad Sci USA 1979;76:2799-2802

11. Lohka MJ, Hayes MK, Maller JL: Purification of maturationpromoting factor, an intracellular regulator of early mitotic events. Proc Natl Acad Sci USA 1988;85:3009-3013

12. Yamashita S, Maller JL: Identification of an activator required for elevation of maturation-promoting factor(MPF) activity by $\gamma$-s-ATP. J Cell Biol 1990;110:1583-1588

13. Kimura Y, Yanagimachi R: Intracytoplasmic sperm injection in the mouse. Biol Reprod 1995; 52:709-720

14. Van de Wiel DFM, Bar-Ami S, Tsafriri A, et al.: Oocyte maturation inhibitor, inhibin and steroid concentrations in porcine follicular fluid at various stages of the estrous cycle. J Reprod Fertil 1983;68:247-252

15. Cho WK, Stern S, Biggers JD: Inhibitory effect of dibutyryl cAMP on mouse oocyte maturation in vitro. J Exp Zool 1974; $187: 383-386$

16. Lindner HR, Tsafriri A, Lieberman ME, et al.: Gonadotropin action on cultured Graafian follicles: Induction of maturation division of the mammalian oocyte and differentiation of the luteal cell. Recent Prog Horm Res 1974;30:79-138

17. Dekel N, Galiani D, Sherizly I: Dissociation between the inhibitory and the stimulatory action of cAMP on maturation of rat oocytes. Mol Cell Endocrinol 1988;56:115-121

18. Cross PC: The role of cumulus cells and serum in mouse oocyte maturation in vitro. J Reprod Fertil 1973;34:241-245

19. Gavin AC, Vassalli JD, Cavadore JC, Schorderet-Slatkine S: Okadaic acid and p13suc1 modulate the reinitiation of meiosis in mouse oocytes. Mol Reprod Dev 1992;33:287-296 